Case Report and Review of the Literature

\title{
Self-Made Minimally Invasive Broken Nail Extractor for Distal Broken Segment of Cannulated Intramedullary Nails: One Case Application Report and Literature Review
}

\author{
Changbao Wei ${ }^{1}$, Qudong Yin ${ }^{1 *}$, Guo Shu $\mathrm{Ma}^{2}$, Sanjun Gu${ }^{1}$ and Yu Liu ${ }^{1}$ \\ ${ }^{1}$ Department of Orthopaedics, Wuxi No. 9 People's Hospital Affiliated to Soochow University, Jiangsu, China \\ ${ }^{2}$ Department of Orthopedics and Traumatology, Wuxi No. 2 Traditional Chinese Medicine Hospital, Jiangsu, China
}

A R T I C L E I N F O
Article history:
Received: 27 December, 2021
Accepted: 10 January, 2022
Published: 21 January, 2022
Keywords:
Broken intramedullary nail
broken nail extractor
minimally invasive
self-made

\section{A R T I C L E I N F O}

Article history:

Accepted: 10 January, 2022

Published: 21 January, 2022

Keywords:

Broken intramedullary nail

broken nail extractor

self-made

\begin{abstract}
A B S T R A C T
Introduction: Successful minimally invasive extraction of a broken intramedullary distal piece of the nail is very challenging. We herein report a simple and reproducible technique of extraction of broken cannulated intramedullary nails using a self-made minimally invasive broken nail extractor.

Case Presentation: We present a 48-year-old male patient who was admitted to our hospital for internal implant removal, whose right tibial fracture had healed, and a broken intramedullary nail was retained at the distal of the tibial for 12 years. The preoperative X-ray films showed the broken intramedullary nail was cannulated and the outer diameter is $10 \mathrm{~mm}$, larger than the isthmus of the medullary cavity.

Results: After reaming with $\Phi 11 \mathrm{~mm}$ drill, the self-made broken nail extractor was inserted into the medullary cavity from the nail entry point and passed through the canal of the broken nail, and successfully pulled out the broken nail.

Conclusion: Our technique is a simple, reproducible alternative that has a high extraction success rate for the distal broken segments of cannulated intramedullary nails.
\end{abstract}

